\title{
ESTUDO EXPERIMENTAL E MODELAGEM MATEMÁTICA DA COPOLIMERIZAÇÃO DE ÁCIDO ACRÍLICO COM TRIMETILOLPROPANO TRIACRILATO INCLUINDO O PERÍODO DE PÓS-GELIFICAÇÃO
}

\author{
L. G. AGUIAR, E. F. SOUZA, R. GIUDICI \\ Escola Politécnica da Universidade de São Paulo, Dep. Engenharia Química \\ E-mail para contato: rgiudici@usp.br
}

\begin{abstract}
RESUMO - Neste estudo, foram realizadas copolimerizações de ácido acrílico com o reticulante trimetilolpropano triacrilato (TMPTA) em solução aquosa a $50^{\circ} \mathrm{C}$, utilizandose persulfato de sódio como iniciador. Foram avaliados os perfis de conversão de monômero e fração de gel em função da quantidade de reticulante adicionado. Foi desenvolvido um modelo matemático baseado em balanços de espécies, método dos momentos e fracionamento numérico para representar o sistema em questão. Bom ajuste foi obtido entre o modelo e os dados experimentais tanto antes quando após a gelificação. O modelo leva em conta as reações de ciclização através de um balanço de sequências contidas nas cadeias de polímero. As simulações foram conduzidas fazendo-se uso de 3 gerações de cadeias poliméricas (fracionamento numérico) e as ciclizações foram consideradas para cadeias primárias contendo um máximo de 100 unidades monoméricas.
\end{abstract}

\section{INTRODUÇÃO}

Hidrogéis são cadeias tridimensionais de polímeros ou copolímeros hidrofílicos que, apesar da sua afinidade com a água, são insolúveis devido à presença de ligações cruzadas na sua estrutura. Outra característica dos hidrogéis é que, devido as suas propriedades físico-químicas, apresentam sensibilidade a estímulos externos, como por exemplo, à temperatura e ao pH.

Devido a estas características, os hidrogéis têm sido utilizados no dia-a-dia em diferentes formas, dependendo da aplicação necessária, por exemplo, em cremes dentais, gel de cabelo, lentes de contato e em muitos outros produtos das indústrias farmacêutica e cosmética. Na indústria farmacêutica o avanço mais significativo tem sido em relação à liberação controlada de medicamentos, área a qual os hidrogéis se destacam devido a sua elevada biocompatibilidade e estrutura de rede ajustável para controlar a difusão da droga (SIMÕES, S.; FIQUEIRAS, A., 2012). Por exemplo, os hidrogéis para administração por via oral de fármacos apresentam sensibilidade ao $\mathrm{pH}$, deste modo respondem à variação de $\mathrm{pH}$ no trato-gastrointestinal, podendo proporcionar uma sistema de liberação não invasivo para algumas drogas intravenosas, tais como a insulina (HOARE; KOHANE, 2008; RASOOL; YASIN; HENG; AKHTER, 2010). Tais hidrogéis são sensíveis ao pH devido aos íons provenientes dos grupos de ácido carboxílico presentes em sua estrutura química. No 
entanto, a copolimerização de monômeros acrílicos com polivinílicos em fase aquosa apresenta uma cinética complexa e não totalmente compreendida.

Uma característica importante da polimerização do ácido acrílico em solução aquosa é que a constante de velocidade de propagação é fortemente afetada pelo meio reacional. Devido à falta de compreensão mecanicista deste processo, a literatura ainda apresenta informações escassas referentes às velocidades das reações envolvidas na polimerização de hidrogéis. Deste modo, estudos têm sido realizados para se entender a influência de fatores como temperatura e $\mathrm{pH}$ na velocidade de reação destes sistemas químicos. KABANOV et al (1975) encontrou uma dependência não linear entre a taxa global de polimerização e o pH e atribuiu este comportamento à formação de pares de ions compostos por unidades terminais ionizadas de cadeias em crescimento e cátions presentes no meio reacional.

Os estudos cinéticos de copolimerização de ácido acrílico, com o monômero trivinílico TMPTA são relatados na literatura (ARRIOLA; CUTIE; HENTON; POWELL; SMITH, 1997; SCOTT; PEPPAS, 1997). GONÇALVES et al. (2011) também estudaram esse sistema e desenvolveram um modelo cinético baseado em equações de balanços populacionais e funções geradoras para representar o processo. No entanto, este modelo negligencia as reações de ciclização intramolecular, sendo mais realista para quantidades pequenas de agente de reticulação.

Com isso, o presente trabalho tem como objetivo descrever uma abordagem alternativa para a copolimerização de ácido acrílico / TMPTA com base no método de momentos e no fracionamento numérico. Dados experimentais foram obtidos em laboratório a fim de validar o modelo.

\section{MATERIAIS E MÉTODOS}

\subsection{Parte Experimental}

Hidrogéis de ácido acrílico foram produzidos através da copolimerização por radicais livres em solução aquosa utilizando diferentes concentrações do agente reticulante trimetilolpropano triacrilato. As reações foram realizadas em tubos de ensaio a uma temperatura de $50{ }^{\circ} \mathrm{C}$. Após misturar todos os reagentes, quantidades conhecidas da solução resultante foram adicionadas em tubos de ensaio. Cada reação foi realizada com uma razão mássica de ácido acrílico/água de 0,3 e uma

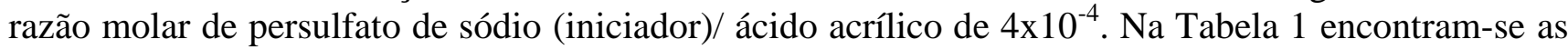
frações molares de agente reticulante (TMPTA) em relação à mistura monomérica para cada hidrogel produzido $\left(\mathrm{Y}_{\mathrm{c}}\right)$.

Tabela 1 - Fração molar de TMPTA em cada reação.

\begin{tabular}{cl}
\hline Reação & $\mathbf{Y}_{\mathbf{C}}(\boldsymbol{\%})$ \\
\hline XA1 & 0,00 \\
XA2 & 0,05 \\
XA3 & 0,13 \\
XA4 & 0,25 \\
\hline
\end{tabular}




\subsection{Modelagem Matemática}

Na Tabela 2 encontra-se a relação dos passos do mecanismo reacional considerado na produção de hidrogéis de ácido acrílico através da polimerização por radicais livres.

Tabela 2 - Reações consideradas no modelo.

\begin{tabular}{lcc}
\hline \multicolumn{1}{c}{ Nome da Reação } & Equação Química & Observação \\
\hline Decomposição do iniciador & \multicolumn{1}{c}{$\stackrel{\mathrm{k}_{\mathrm{d}}}{\rightarrow} 2 \mathrm{R}_{0}$} & - \\
Iniciação do monômero & $\mathrm{R}_{0}+\mathrm{M}_{\mathrm{j}} \stackrel{\mathrm{k}_{\mathrm{Ij}}}{\rightarrow} \mathrm{R}_{1, \mathrm{j}}$ & $1 \leq \mathrm{j} \leq 2$ \\
Propagação do monômero & $\mathrm{R}_{\mathrm{r}, \mathrm{i}}+\mathrm{M}_{\mathrm{j}} \stackrel{\mathrm{k}_{\mathrm{p}, \mathrm{ij}}}{\rightarrow} \mathrm{R}_{\mathrm{r}+1, \mathrm{j}}$ & $1 \leq \mathrm{i} \leq 5 \mathrm{e} 1 \leq \mathrm{j} \leq 2$ \\
Iniciação das duplas ligações pendentes & $\mathrm{R}_{0}+\mathrm{D}_{\mathrm{i}} \stackrel{\mathrm{k}_{\mathrm{I}, \mathrm{i}}}{\rightarrow} \mathrm{R}_{\mathrm{r}, \mathrm{i}}$ & $3 \leq \mathrm{i} \leq 4$ \\
Propagação das duplas ligações pendentes & $\mathrm{R}_{\mathrm{r}, \mathrm{j}}+\mathrm{D}_{\mathrm{i}} \stackrel{\mathrm{k}_{\mathrm{p}, \mathrm{ij}}}{\rightarrow} \mathrm{R}_{\mathrm{s}, \mathrm{i}}$ & $1 \leq \mathrm{j} \leq 5 \mathrm{e} 3 \leq \mathrm{i} \leq 4$ \\
Transferência de cadeia para o polímero & $\mathrm{P}_{\mathrm{S}}+\mathrm{R}_{\mathrm{r}, \mathrm{i}} \stackrel{\mathrm{k}_{\mathrm{fr}, \mathrm{i}}}{\rightarrow} \mathrm{R}_{\mathrm{s}, 5}+\mathrm{P}_{\mathrm{r}}$ & $1 \leq \mathrm{i} \leq 5$ \\
Abstração de hidrogênio & $\mathrm{P}_{\mathrm{S}}+\mathrm{R}_{0} \stackrel{\mathrm{k}_{\mathrm{h}}}{\rightarrow} \mathrm{R}_{\mathrm{s}, 5}+\mathrm{S}$ & - \\
Inibição & $R_{r, i}+Z \stackrel{k_{z}}{\rightarrow} P_{r}$ & $0 \leq \mathrm{i} \leq 5$ \\
Terminação por combinação & $\mathrm{R}_{\mathrm{r}, \mathrm{j}}+\mathrm{R}_{\mathrm{s}, \mathrm{i}} \stackrel{\mathrm{k}_{\mathrm{tc}}}{\rightarrow} \mathrm{P}_{\mathrm{r}+\mathrm{s}}$ & $1 \leq \mathrm{j} \leq 5 \mathrm{e} 1 \leq \mathrm{i} \leq 5$ \\
Terminação por desproporcionamento & $\mathrm{R}_{\mathrm{r}, \mathrm{j}}+\mathrm{R}_{\mathrm{s}, \mathrm{i}} \stackrel{\mathrm{k}_{\mathrm{td}}}{\rightarrow} \mathrm{P}_{\mathrm{r}}+\mathrm{P}_{\mathrm{s}}$ & $1 \leq \mathrm{j} \leq 5$ e $1 \leq \mathrm{i} \leq 5$ \\
\hline
\end{tabular}

'i' e 'j' representam os tipos de monômero, radical e dupla ligação pendente nas cadeias poliméricas. ' $r$ ' e 's' representam o tamanho genérico de uma cadeia polimérica.

O modelo apresentado destaca-se por levar em conta as reações de ciclização, as quais foram consideradas partir da análise das sequências ao longo do processo, como relatado em trabalho anterior (AGUIAR et al., 2014). No presente modelo foram consideradas as seguintes hipóteses e aproximações:

- Estado pseudo-estacionário para os radicais (PSSH);

- Pseudo-homopolimerização;

- Cada cadeia de polímero solúvel possui somente um centro radicalar (monorradical);

- Fechamento do sistema de equações dos momentos através da relação de Saidel e Katz (1968):

$$
Q_{i 3}=\frac{2 Q_{i 2}{ }^{2}}{Q_{i 1}}-\frac{Q_{i 2} Q_{i 1}}{Q_{i 0}}
$$

- Durante cada passo de integração, as frações de cada tipo de radical são constantes;

- A velocidade das reações bimoleculares envolvendo grupos poliméricos é independente do tamanho da cadeia de polímero;

- A velocidade de ciclização ocorre em sequências com 2 ou mais unidades poliméricas; 


\section{9 a 22 de outubro de 2014 \\ Florianópolis/SC}

- A taxa de ciclização passa a ser desprezível em sequências contendo mais do que 100 unidades monoméricas $\left(\mathrm{r}_{\text {máx }}\right)$;

- Somente cadeias primárias podem ciclizar;

- A formação de novas sequências resultantes de uma ciclização não é contabilizada no modelo;

- A ciclização só ocorre através de propagação cíclica;

- A redução da constante cinética de ciclização com o aumento do comprimento da sequência segue a equação de Rolfes e Stepto (1990):

$$
k_{P i j, r}^{C}=\left[\frac{1}{(r-1)}\right]^{1,5} k_{P i j, 2}^{C}
$$

O modelo construído para a realização das simulações foi desenvolvido tendo em conta os seguintes métodos: balanços de espécies, grupos poliméricos e sequências; método dos momentos e fracionamento numérico. O método dos momentos contabiliza os diferentes tamanhos de cadeia, eliminando a necessidade de desenvolver balanços molares para cada um. O método do fracionamento numérico (TEYMOUR; CAMPBELL, 1994) foi utilizado com o objetivo de se prever o comportamento do processo no período pós-gelificação.

\section{RESULTADOS E DISCUSSÕES}

O modelo foi validado com os dados obtidos experimentalmente. Nas figuras 1 e 2 são apresentados os resultados de simulação para conversão e fração de gel respectivamente. As simulações foram realizadas com os seguintes valores de parâmetros: $\mathrm{k}_{\mathrm{p} 11}=6000 ; \mathrm{k}_{\mathrm{p} 12}=7792 ; \mathrm{k}_{\mathrm{p} 13}=$ 1200; $\mathrm{k}_{\mathrm{p} 14}=600$; razões de reatividade $\mathrm{r}_{2}=\mathrm{k}_{\mathrm{p} 2 \mathrm{i}} / \mathrm{k}_{\mathrm{pli}}=1, \mathrm{r}_{3}=0,5, \mathrm{r}_{4}=0,24, \mathrm{r}_{5}=1 ; \mathrm{k}_{\mathrm{d}}=7,42 \times 10^{-6} ; \mathrm{k}_{\mathrm{Ij}}=\mathrm{k}_{\mathrm{p} 1 \mathrm{j}}$; $\mathrm{k}_{\mathrm{fr}}=\mathrm{k}_{\mathrm{h}}=\mathrm{k}_{\mathrm{td}}=0 ; \mathrm{k}_{\mathrm{z}}=8 \times 10^{5} ; \mathrm{k}_{\mathrm{tc}}=5 \times 10^{7}$, eficiência do iniciador $\mathrm{f}=0,7$ e constante de ciclização para radical com 2 unidades monoméricas $\mathrm{k}_{\mathrm{p} 13,2}^{\mathrm{c}}=8,5 \times 10^{3}$. As constantes cinéticas foram obtidas por tentativa e erro fazendo-se uma comparação visual do modelo com os dados experimentais, considerando-se uma temperatura de $50^{\circ} \mathrm{C}$. Todas as unidades estão em $\mathrm{L} /\left(\right.$ mol.s) exceto as de $\mathrm{k}_{\mathrm{d}} \mathrm{e}$ $\mathrm{k}_{\mathrm{p} 13,2}^{\mathrm{c}}$ que estão s${ }^{-1}$. Nota-se que os perfis experimentais de conversão situam-se em uma mesma faixa, com pouca variação entre os mesmos. Isto ocorre, pois, pequenas quantidades de TMPTA foram adicionadas aos experimentos de modo a não afetar significativamente o perfil de conversão. Os dados experimentais foram obtidos através de gravimetria. A dispersão observada nos perfis experimentais está associada a imprecisões da balança analítica utilizada. Observa-se que o perfil de conversão para XA1 está ligeiramente diferente dos demais. Em teoria, os perfis deveriam ser semelhantes em função das formulações, o que sugere que esta discrepância esteja associada a algum erro de manuseio ou imprecisão da balança. Durante o preparo dos experimentos, oxigênio do ar pode ter sido dissolvido no meio reacional antes de os tubos terem sido fechados, causando inibição de radicais no início da reação. Na Tabela 3 encontram-se os valores das concentrações iniciais de oxigênio que foram consideradas nas simulações. A discrepância observada entre as previsões da conversão pelo modelo se deve às diferentes concentrações de oxigênio dissolvido durante as simulações. A presença do mecanismo de inibição no modelo permite explicar o período de indução inicial observado nos dados experimentais até cerca de 40 minutos de reação. 


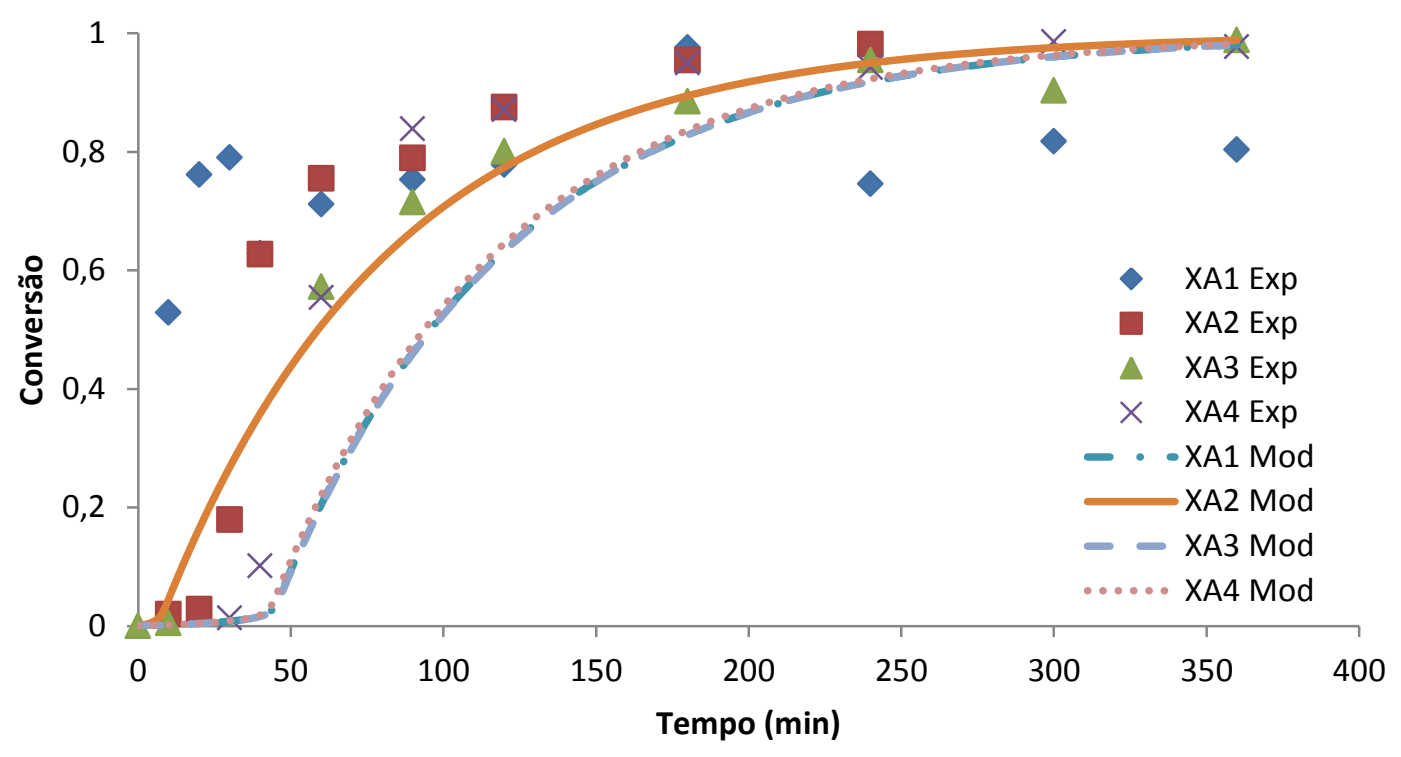

Figura 1 - Resultados de conversão. Exp: Dados experimentais, MOD: Previsão do modelo.

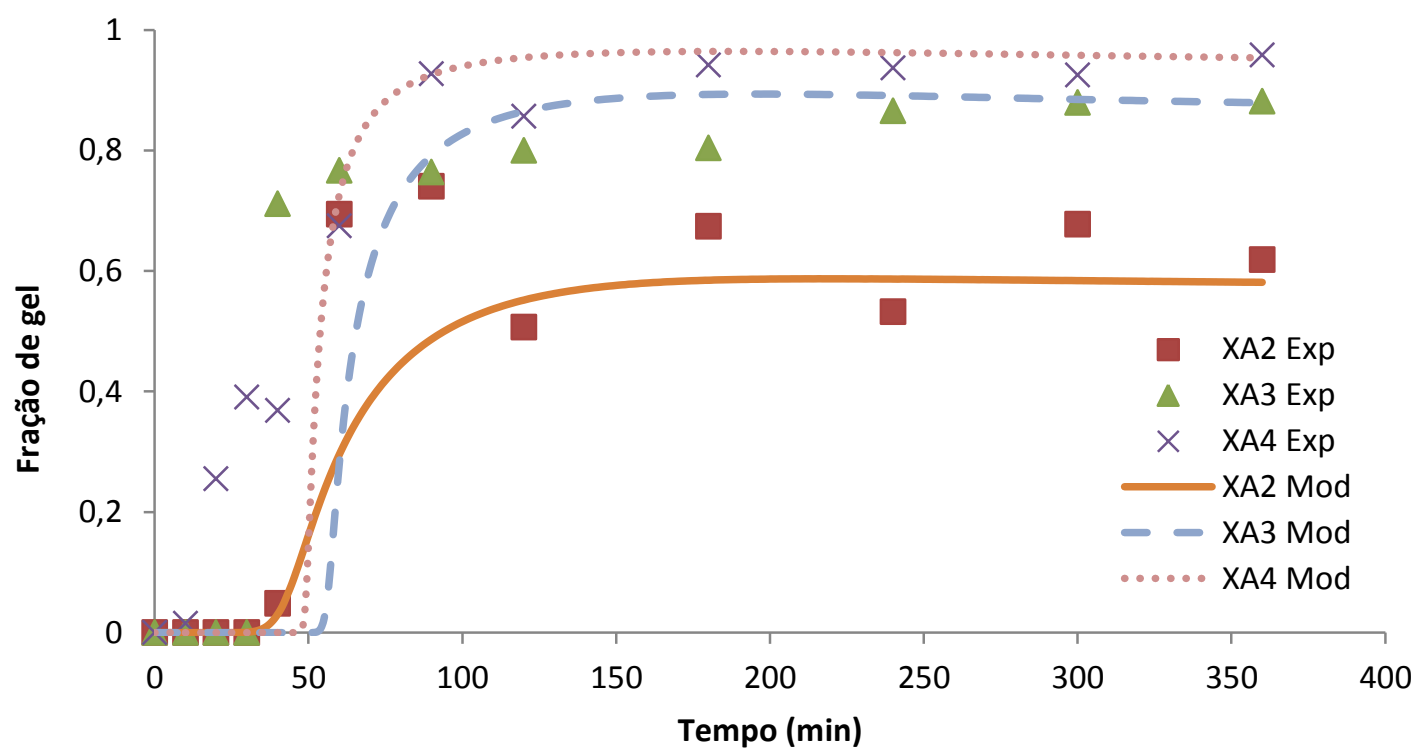

Figura 2 - Resultados de fração de gel. Exp: Dados experimentais, MOD: Previsão do modelo. 
Tabela 3 - Concentração inicial de $\mathrm{O}_{2}$ (Inibidor).

\begin{tabular}{cc}
\hline Reação & Concentração de $\mathbf{O}_{\mathbf{2}}$ dissolvido $\left(\mathbf{m o l}^{-L^{-1}}\right)$ \\
\hline XA1 & $1,80 \times 10^{-4}$ \\
XA2 & $3,20 \times 10^{-5}$ \\
XA3 & $1,80 \times 10^{-5}$ \\
XA4 & $1,80 \times 10^{-5}$ \\
\hline
\end{tabular}

Na Figura 2, observa-se que quanto maior a quantidade de reticulante, maior é a fração de gel experimental obtida para o mesmo período de tempo. Isto ocorre porque, quanto maior for a quantidade de reticulante presente no meio reacional, maior será o número de ligações cruzadas formadas, o que levará a uma aceleração da gelificação. O modelo foi capaz de prever razoavelmente os perfis experimentais de fração de gel.

A incidência das reações de ciclização em copolimerizações que apresentam um sistema reticulado tem sido notada principalmente quando se obtém um tempo de gelificação experimental maior do que se esperava teoricamente ao se desconsiderar tal reação. A ciclização é uma reação importante a ser considerada no mecanismo, principalmente no que diz respeito ao consumo de duplas ligações pendentes no início da reação (cadeias mais afastadas). Quanto maior o comprimento de uma sequência conectando um centro radicalar a uma dupla ligação pendente, menor é a probabilidade de ocorrer a ciclização primária com esta sequência. $\mathrm{O}$ ajuste obtido nas figuras 1 e 2 foi possível considerando-se $\mathrm{k}_{\mathrm{p} 13,2}^{\mathrm{c}}=8,5 \times 10^{3} \mathrm{~s}^{-1}$. Para uma simulação com o mesmo modelo, considerando-se ausência de ciclizações, as previsões de fração de gel são consideravelmente superestimadas.

Para a obtenção dos perfis de conversão e fração de gel considerou-se uma constante de propagação $\left(\mathrm{k}_{\mathrm{p} 11}\right)$ igual a $6000 \mathrm{~L} \cdot \mathrm{mol}^{-1} \mathrm{~s}^{-1}$, que está próxima do valor da constante de propagação para homopolimerização bulk de ácido acrílico à uma temperatura de $50{ }^{\circ} \mathrm{C}$ (GONÇALVES et al. 2011). Neste modelo, os perfis foram gerados assumindo a existência de 3 gerações de cadeias (fracionamento numérico). Ao se utilizar valores maiores para o número de gerações, o modelo passa a apresentar limitações relacionadas aos integradores do MATLAB para alguns casos estudados. Isto se dá devido ao aumento da rigidez do sistema de equações diferenciais ordinárias, pois gerações maiores comportam cadeias poliméricas maiores, as quais apresentam concentrações muito baixas. Esta limitação está associada ao passo de integração do MATLAB, que não pode ser definido abaixo de um determinado limite, mesmo com o uso dos integradores stiff disponibilizados pelo MATLAB. Porém, o método do fracionamento numérico não apresenta limite de gerações. Em teoria, quanto maior for o número de gerações, mais próximo o modelo se situará da realidade. 


\section{CONCLUSÕES}

Um modelo matemático para a copolimerização de ácido acrílico com TMPTA incluindo o período pós-gelificação e as reações de ciclização foi desenvolvido. O modelo forneceu boas previsões de conversão de monômero e fração de gel para reações conduzidas a $55^{\circ} \mathrm{C}$. Apesar de as reações terem sido realizadas em solução aquosa, obteve-se, através de ajuste de parâmetros, uma constante de propagação de ácido acrílico no valor de $6000 \mathrm{~L} \cdot \mathrm{mol}^{-1} \mathrm{~s}^{-1}$, que se situa próxima a valores publicados na literatura para polimerizações bulk. Observou-se que quanto maior a quantidade de TMPTA, maior é a fração de gel obtida experimentalmente. No modelo, também foi importante considerar a concentração de oxigênio, que apresentou influencia sobre os perfis de conversão e fração de gel. Em suma, através deste trabalho pode-se concluir que o modelo matemático desenvolvido para a copolimerização do ácido acrílico com TMPTA é capaz de representar as principais características deste sistema.

\section{NOMENCLATURA}

\begin{tabular}{|c|c|}
\hline I & Iniciador \\
\hline $\mathbf{D}_{\mathbf{i}}$ & Dupla ligação pendente do tipo "i”" \\
\hline $\mathbf{k}_{\mathbf{d}}$ & Constante cinética de decomposição do iniciador $\left(\mathrm{s}^{-1}\right)$ \\
\hline $\mathbf{k}_{\mathbf{f r}, \mathbf{j}}$ & Constante cinética de transferência de cadeia de um radical do tipo "i" (L.mol $\left.{ }^{-1} \cdot \mathrm{s}^{-1}\right)$ \\
\hline $\mathbf{k}_{\mathbf{h}}$ & Constante cinética da reação de abstração de hidrogênio $\left(\mathrm{L} \cdot \mathrm{mol}^{-1} \cdot \mathrm{s}^{-1}\right)$ \\
\hline $\mathbf{k}_{\mathbf{I j}}$ & Constante cinética da reação de iniciação do monômero ou do reticulante ( $\left.\mathrm{L} \cdot \mathrm{mol}^{-1} \cdot \mathrm{s}^{-1}\right)$ \\
\hline $\mathbf{k}_{\mathbf{p}, \mathbf{i j}}$ & $\begin{array}{l}\text { Constante cinética da reação de propagação envolvendo um radical do tipo "i" e o } \\
\text { monômero do tipo "j" }\end{array}$ \\
\hline $\mathbf{k}_{\mathrm{tc}}$ & Constante cinética da reação de terminação por combinação $\left(\mathrm{L} \cdot \mathrm{mol}^{-1} \cdot \mathrm{s}^{-1}\right)$ \\
\hline $\mathbf{k}_{\mathbf{t d}}$ & Constante cinética da reação de terminação por dismutação $\left(\mathrm{L} \cdot \mathrm{mol}^{-1} \cdot \mathrm{s}^{-1}\right)$ \\
\hline $\mathbf{k}_{\mathbf{z}}$ & Constante de inibição $\left(\mathrm{L} \cdot \mathrm{mol}^{-1} \cdot \mathrm{s}^{-1}\right)$ \\
\hline $\mathbf{M}_{\mathbf{j}}$ & Monômero do tipo j (j=1: Ácido Acrílico, j=2: Trimetilpropano Triactilato) \\
\hline $\mathbf{P}_{\mathbf{r}}$ & Polímero morto contendo "r" unidades monoméricas \\
\hline $\mathbf{P}_{\mathbf{r}, \mathbf{i}}$ & $\begin{array}{l}\text { Polímero morto contendo duplas ligações pendentes do tipo "i" e "r" unidades } \\
\text { monoméricas }\end{array}$ \\
\hline $\mathbf{P}_{\mathbf{r}+\mathbf{s}}$ & Polímero morto contendo "r+s" unidades monoméricas \\
\hline $\mathbf{P}_{\mathrm{s}}$ & Polímero morto contendo "s" unidades monoméricas \\
\hline $\mathbf{R}_{\mathbf{0}}$ & Radical Primário \\
\hline $\mathbf{R}_{\mathbf{r}, \mathbf{j}}$ & Radical polimérico de tamanho "r" e do tipo "j" $(j=1 ; j=2 ; j=3 ; j=4 ; j=5)$ \\
\hline $\mathbf{R}_{\mathrm{s}}$ & Radical polimérico do tipo "s" \\
\hline $\mathbf{S}$ & Espécie inerte \\
\hline $\mathbf{Z}$ & Inibidor \\
\hline
\end{tabular}

\section{REFERÊNCIAS}

AGUIAR, L. G.; GONÇALVES; M. A. D., PINTO; V. D., DIAS, R. C. S.; COSTA, M. R. P. F. N.; GIUDICI, R. (2014). Development of Cyclic Propagation Kinetics for Modeling the 
Nitroxide-mediated Radical Copolymerization of Styrene-Divinylbenzene. Macromolecular Reaction Engineering, 8(4), 282-294. doi:10.1002/mren.201300105

ARRIOLA, D. J.; CUTIE, S. S.; HENTON, D. E.; POWELL, C.; SMITH, P. B. (1997). Crosslinker reactivity and the structure of superabsorbent gels. J. Appl. Polym. Sci, 63, 439451.

GONÇALVES, M. A. D.; PINTO, V. D.; DIAS, R. C. S.; COSTA, M. R. P. F. N. (2011). Modeling Studies on the Synthesis of Superabsorbent Hydrogels Using Population Balance Equations. Macromolecular Symposia, 306-307(1), 107-125. doi:10.1002/masy.201000151

HOARE, T. R.; KOHANE, D. S. (2008). Hydrogels in drug delivery: Progress and challenges. Polymer, 49(8), 1993-2007. doi:10.1016/j.polymer.2008.01.027

KABANOV, V. A.; TOPCHIEV, D. A.; KARAPUTADZE, T. M.; MKRTCHIAN, L. A. (1975). Kinetics and mechanism of radical polymerization of weak unsaturated acids in aqueous solutions. European Polymer Journal, 11(2), 153-159. doi:10.1016/0014-3057(75)90140-8

RASOOL, N.; YASIN, T.; HENG, J. Y. Y.; AKHTER, Z. (2010). Synthesis and characterization of novel $\mathrm{pH}$-, ionic strength and temperature- sensitive hydrogel for insulin delivery. Polymer, 51(8), 1687-1693. doi:10.1016/j.polymer.2010.02.013

ROLFES, H.; STEPTO, R. (1990). Network formation and properties: rate theory description of effects of ring formation on elastic shear modulus of RA2+RA3 networks. Macromolecular Symposia, 40, 61-79.

SAIDEL, G. M.; KATZ, S. (1968). Dynamic analysis of branching in radical polymerization. Journal of Polymer Science Part A-2: Polymer Physics, 6(6), 1149-1160. doi:10.1002/pol.1968.160060608

SCOTT, R. A.; PEPPAS, N. A. (1997). Kinetic study of acrylic acid solution polymerization. AIChE Journal, 43(1), 135-144. doi:10.1002/aic.690430116

SIMÕES S.; FIQUEIRAS A. V. F. (2012). Modular Hydrogels for Drug Delivery. Journal of Biomaterials and Nanobiotechnology, 3, 185-199.

TEYMOUR, F.; CAMPBELL, J. D. (1994). Analysis of the Dynamics of Gelation in Polymerization Reactors Using the "Numerical Fractionation" Technique. Macromolecules, 27(9), 2460-2469. doi:10.1021/ma00087a015 